\title{
Discovery of breast cancer risk genes and establishment of a prediction model based on estrogen metabolism regulation
}

Feng Zhao ${ }^{1,2 \dagger}$, Zhixiang Hao ${ }^{1 \dagger}$, Yanan Zhong ${ }^{1}$, Yinxue Xu', Meng Guo ${ }^{3}$, Bei Zhang ${ }^{4}$, Xiaoxing Yin ${ }^{1}$, Ying $\mathrm{Li}^{3}$ and Xueyan Zhou ${ }^{1 *}$ id

\begin{abstract}
Background: Multiple common variants identified by genome-wide association studies have shown limited evidence of the risk of breast cancer in Chinese individuals. In this study, we aimed to uncover the relationship between estrogen levels and the genetic polymorphism of estrogen metabolism-related enzymes in breast cancer (BC) and establish a risk prediction model composed of estrogen-metabolizing enzyme genes and GWAS-identified breast cancer-related genes based on a polygenic risk score.

Methods: Unrelated BC patients and healthy subjects were recruited for analysis of estrogen levels and single nucleotide polymorphisms (SNPs) in genes encoding estrogen metabolism-related enzymes. The polygenic risk score (PRS) was used to explore the combined effect of multiple genes, which was calculated using a Bayesian approach. An independent sample t-test was used to evaluate the differences between PRS scores of BC and healthy subjects. The discriminatory accuracy of the models was compared using the area under the receiver operating characteristic (ROC) curve.
\end{abstract}

Results: The estrogen homeostasis profile was disturbed in $B C$ patients, with parent estrogens (E1, E2) and carcinogenic catechol estrogens (2/4-OHE1, 2-OHE2, 4-OHE2) significantly accumulating in the serum of BC patients. We then established a PRS model to evaluate the role of SNPs in multiple genes. PRS model 1 (M1) was established from SNPs in 6 GWAS-identified high risk genes. On the basis of M1, we added SNPs from 7 estrogen metabolism enzyme genes to establish PRS model 2 (M2). The independent sample t-test results showed that there was no difference between $B C$ and healthy subjects in $M 1(P=0.17)$; however, there was a significant difference between $\mathrm{BC}$ and healthy subjects in $\mathrm{M} 2\left(P=4.9^{*} 10^{-5}\right)$. The ROC curve results showed that the accuracy of $\mathrm{M} 2(\mathrm{AUC}=$ $62.18 \%$ ) in breast cancer risk identification was better than that of M1 (AUC $=54.56 \%$ ).

(Continued on next page)

\footnotetext{
* Correspondence: zxy851107@126.com

${ }^{\dagger}$ Feng Zhao and Zhixiang Hao contributed equally to this work.

'Jiangsu Key Laboratory of New Drug Research and Clinical Pharmacy,

College of Pharmacy, Xuzhou Medical University, 209 Tongshan Road,

Xuzhou 221004, China

Full list of author information is available at the end of the article
}

(c) The Author(s). 2021 Open Access This article is licensed under a Creative Commons Attribution 4.0 International License, which permits use, sharing, adaptation, distribution and reproduction in any medium or format, as long as you give appropriate credit to the original author(s) and the source, provide a link to the Creative Commons licence, and indicate if changes were made. The images or other third party material in this article are included in the article's Creative Commons licence, unless indicated otherwise in a credit line to the material. If material is not included in the article's Creative Commons licence and your intended use is not permitted by statutory regulation or exceeds the permitted use, you will need to obtain permission directly from the copyright holder. To view a copy of this licence, visit http://creativecommons.org/licenses/by/4.0/ The Creative Commons Public Domain Dedication waiver (http://creativecommons.org/publicdomain/zero/1.0/) applies to the data made available in this article, unless otherwise stated in a credit line to the data. 
(Continued from previous page)

Conclusion: Estrogen and related metabolic enzyme gene polymorphisms are closely related to BC. The model constructed by adding estrogen metabolic enzyme gene SNPs has a good predictive ability for breast cancer risk, and the accuracy is greatly improved compared with that of the PRS model that only includes GWAS-identified gene SNPS.

Keywords: Breast cancer, Risk prediction, Estrogens, Estrogen-metabolizing enzyme, Gene polymorphism, Polygenic risk score

\section{Background}

Breast cancer is the most common malignant disease among women worldwide, accounting for $24 \%$ of new cancer cases and $15 \%$ of cancer deaths in 2018 , and incident cases are expected to increase by more than $46 \%$ by 2040, according to the GLOBOCAN Cancer Tomorrow prediction tool, which will seriously endanger women's lives and health [1]. At present, people's understanding of breast cancer is deepening substantially, and new treatment strategies for tumors, including breast cancer, are continually emerging $[2,3]$. With continuous improvements in diagnosis and treatment methods, the survival rate of breast cancer patients has been greatly improved. Early prediction, early detection, and early treatment of high-risk groups are the key issues that urgently need to be solved in the clinic.

The occurrence and development of breast cancer are closely related to genetic and environmental factors. In 1989, Gail proposed the breast cancer risk prediction model, which included factors such as age at evaluation, age at menarche, age at first live birth, race, number of breasts, and family history of breast cancer [4, 5]. Some subsequent prediction models also involved BRCA1/2, estrogen replacement therapy, mammography screening times, and genetic polymorphisms. Rare high-risk mutations, particularly in the BRCA1 and BRCA2 genes, explain less than $20 \%$ of the twofold familial relative risk (FRR) and account for a small proportion of breast cancer cases in the general population. Low-frequency variants conferring intermediate risk, such as those in CHEK2, ATM, and PALB2, explain 2 to $5 \%$ of the FRR [6]. Genome-wide association studies (GWASs) have led to the discovery of multiple common, low-risk variants (single nucleotide polymorphisms [SNPs]) associated with breast cancer risk [7]. Recently, it was found that genetic risk factors can account for $31 \%$ of breast cancer risk evaluations [8], which indicates that breast cancer is a multifactorial disease and that genetic factors are important etiological factors involved in the occurrence and development of breast cancer. At present, an increasing number of researchers are inclined to develop a comprehensive genetic risk scoring method to evaluate the polygenic effects of single nucleotide polymorphisms (SNPs) based on GWASs [9-11]. Some well-known studies, such as Mavaddat et al., used 77 GWAS-selected SNPs to construct a PRS for BC. Compared with middle quintile polygenic scores, the risk scores of the highest $1 \%$ were increased threefold [9].

GWASs also have their own limitations. First, a major limitation of genome-wide approaches is the need to adopt a high level of significance to account for multiple tests. Second, GWASs explain only a modest fraction of the missing heritability [12]. Estrogen is an important risk factor for breast cancer. With long-term exposure, super physiological concentrations of estrogen can bind to estrogen receptors, mediate the overexpression of various growth factors, and promote the growth and proliferation of cells, and various metabolites of estrogen can form adducts with DNA, induce genetic mutations and produce direct genotoxicity [13]. Thus, the abnormal accumulation of estrogen and its toxic metabolites in breast tissue is an important risk factor for breast cancer development. Estrogen homeostasis is regulated by estrogen-related metabolic enzymes. Endogenous estrogens are metabolized to be 2-, 4- and 16 $\alpha$-hydroxy estrogens, which are catalyzed by the phase I metabolizing enzymes cytochrome P450 CYP1A1, CYP1B1 and CYP3A4, respectively [14-16]. Hydroxyestrogens are detoxified by conjugation reactions catalyzed by phase II metabolizing enzymes such as COMT, UGTs and SULTs. Thus, the expression level of estrogen and its toxic metabolites can be considered to be a comprehensive reflection of the role of these estrogen metabolic enzymes to a certain extent. Polymorphisms in genes encoding these estrogen-related metabolic enzymes are reported to be closely related to differences in enzyme activities and alter the levels of DNA-damaging species to influence the individual's susceptibility to breast cancer $[14,17,18]$. Genetic epidemiological studies have suggested that there is a correlation between polymorphisms in estrogen metabolism genes and breast cancer risk; however, these results are not consistent [18-20]. This is an important reason for the inconsistency of existing research results that studied the correlation between gene polymorphisms of estrogen metabolic enzymes and breast cancer in isolation. Currently, breast cancer risk gene prediction models have not taken estrogen metabolic enzyme genes into consideration; therefore, further 
optimization is needed from the perspective of overall estrogen metabolism levels.

Based on the above analysis, our research aims to reveal the form of estrogen homeostasis disorders in breast cancer and explore the association between metabolic enzyme gene polymorphisms and breast cancer occurrence from the overall level of estrogen metabolism. Furthermore, we developed a risk score comprising GWAS-selected SNPs and estrogen metabolic enzyme gene SNPs to optimize the breast cancer risk prediction model.

\section{Methods}

\section{Chemicals}

The standards and other chemical reagents were described in our previously published study [21].

\section{Clinical sample collection}

Serum samples were collected during the follicular and luteal phases of 64 premenopausal women (mean age: $45.5 \pm 5.04$ years) first diagnosed with BC and 49 matched healthy women (mean age: $43.7 \pm$ 8.80 years) to detect the level of estrogens. Blood samples were also collected from 140 premenopausal women (mean age: $43.3 \pm 6.24$ years) first diagnosed with $\mathrm{BC}$ and 140 matched healthy women (mean age: $40.2 \pm 3.52$ years) to extract DNA and analyze SNP genotypes. All samples and related data were obtained from the Affiliated Hospital of Xuzhou Medical University, Xuzhou, China, from June 2017 to May 2019. Patients with BC were enrolled from the Department of Nail Surgery, whereas healthy subjects were enrolled from the physical examination center. Blood samples were collected before any therapy.

The enrollment criteria were as follows: no history of smoking; BMI ranging from 19 to 26; and no history of chemotherapy, radiotherapy, or estrogen-related endocrine therapy during blood sample collection. The characteristics of the patients at baseline can be seen in Table 1 . This protocol was approved by the Ethics Committee of the Affiliated Hospital of Xuzhou Medical University. Written informed consent was obtained from each subject before the study.
Quantification of estrogens using the LC-MS/MS method The LC-MS/MS method was performed according to our previously published method [21].

\section{Genotyping analysis}

DNA was extracted from peripheral whole blood with a Tiangen DNA extraction kit (Biotech, Beijing, China). The main metabolic enzymes CYP19A1, CYP1A1, CYP1B1, HSD17B1, COMT, UGTs, and SULTs are involved in the regulation of estrogen metabolism. In this study, according to a previous study and pharmacogenomic database, 1 gene locus that is more common or affects the function and activity of metabolic enzymes was screened from each metabolic enzyme. At the same time, we used GWAS-identified breast cancer-related SNPs according to a previous study [22]. All selected SNPs were potentially functional variants, with minor allelic frequencies (MAFs) of more than 10\%. The allelic discrimination of the following SNPs was performed by SNaPshot assay (Applied Biosystems Inc., Waltham, MA, USA): estrogen metabolic enzyme gene SNPs including CYP19A1 (rs700519), CYP1A1 (rs1048943), CYP1A1 (rs4646903), CYP1B1 (rs1056827), CYP1B1 (rs1056836), COMT (rs4680), HSD17B1 (rs605059), SULT1A1 (rs1042028), and UGT2B7 (rs7439366) and the GWAS-identified high-risk breast cancer gene SNPs including ZNF365 (rs10822013), FGFR2 (rs2981579), RAD51B (rs3784099), TOX3 (rs3803662), MAP3K1 (rs889312), and HCN1 (rs981782). The allelic discrimination analysis was performed by Genesky Biotechnologies Inc., Shanghai, China (http://www.geneskybiotech. com). Detailed information about the basic SNP information can be found in Table 2. To assure genotyping quality, detailed quality control (QC) procedures, including the duplicate identification of genotypes and a Hardy-Weinberg equilibrium (HWE) test, were carried out. All 15 SNPs were successfully genotyped in 280 subjects with call rates of $100 \%$.

\section{Statistical analysis}

SPSS 22.0 software was used to perform statistical analysis. We used the mean \pm SEM to express all estrogen data and Student's t-test to test differences between the two groups. Multivariate analysis was performed using SIMCA 14.0 software.

Table 1 The characteristics of the patients at baseline

\begin{tabular}{lll}
\hline & Patients & Healthy volunteers \\
\hline Age (detecting the level of estrogens) & $45.5 \pm 5.04$ years & $43.7 \pm 8.80$ \\
Age (Analyzing SNP genotype) & $47.61 \pm 3.55$ years & $40.2 \pm 3.52$ years \\
BMl & $24.43 \pm 3.42$ & $23.09 \pm 2.51$ \\
\hline
\end{tabular}

BMI Body mass index 
Table 2 The basic information and HWE testing of each estrogens metabolizing enzymes gene polymorphisms

\begin{tabular}{|c|c|c|c|c|c|c|c|}
\hline Gene & rs number & Chromosome position & Domain & Alleles & Amino acid change & Metabolism estrogens & Test for HWE (p) \\
\hline CYP19A1 & rs700519 & Chr15: 51507968 & exon7 & $\mathrm{G} / \mathrm{A}$ & Arg264Cys & E1(E2) & 0.392 \\
\hline CYP1A1 & rs1048943 & Chr15: 75012985 & exon7 & $\mathrm{T} / \mathrm{C}$ & Ile462Val & 2-OHE1(E2) & 0.241 \\
\hline CYP1A1 & rs4646903 & Chr15: 75011641 & 3'-flanking & $A / G$ & / & 2-OHE1(E2) & 1.000 \\
\hline CYP1B1 & rs1056827 & Chr2: 38302177 & exon2 & $\mathrm{C} / \mathrm{A}$ & Ala119Ser & 4-OHE1(E2) & 0.602 \\
\hline CYP1B1 & rs1056836 & Chr2: 38298203 & exon3 & $\mathrm{G} / \mathrm{C}$ & Val432Leu & 4-OHE1(E2) & 0.101 \\
\hline HSD17B1 & rs605059 & Chr17: 40706906 & exon6 & $\mathrm{G} / \mathrm{A}$ & Gly313Ser & E2 & 0.106 \\
\hline COMT & rs4680 & Chr22: 19951271 & exon4 & $\mathrm{G} / \mathrm{A}$ & Val158Met & 2 (4)-MeOE1(2) & 1.000 \\
\hline SULT1A1 & rs1042028 & Chr16: 28617514 & exon7 & $C / T$ & Arg213His & Sulfated metabolites & 0.144 \\
\hline UGT2B7 & rs7439366 & Chr4: 69964338 & exon2 & $C / T$ & Tyr268His & Glucuronide metabolites & 0.086 \\
\hline ZNF365 & rs10822013 & Chr10: 64251977 & intron4 & $C / T$ & / & / & 0.478 \\
\hline FGFR2 & rs2981579 & Chr10: 123337335 & intron2 & $\mathrm{G} / \mathrm{A}$ & / & / & 0.665 \\
\hline CASC16 & rs3803662 & Chr16: 52586341 & exon4 & $\mathrm{G} / \mathrm{A}$ & / & / & 0.360 \\
\hline RAD51B & rs3784099 & Chr14: 68749927 & intron7 & $\mathrm{G} / \mathrm{A}$ & / & / & 0.456 \\
\hline MAP3K1 & rs889312 & Chr5: 56031884 & / & $\mathrm{A} / \mathrm{C}$ & / & / & 0.776 \\
\hline HCN1 & rs981782 & Chr5: 45285616 & intron6 & $\mathrm{A} / \mathrm{C}$ & / & / & 0.818 \\
\hline
\end{tabular}

HWE Hardy-Weinberg equilibrium

HWE was examined among controls using a goodness-of-fit chi-squared test. The odds ratio (OR) and $95 \%$ confidence interval $(\mathrm{CI})$ were calculated using a logistic regression model to assess the association between the SNPs and the risk of breast cancer.

We established a PRS to estimate the multigene contribution of estrogen-metabolic enzyme gene loci for breast cancer susceptibility, which was created using marginally significant SNPs associated with breast cancer risk based on the per-allele models. For SNPs in strong linkage disequilibrium located on the same gene or chromosome, we chose the one variant with the lowest $P$ value in the per-allele model as a candidate. The basic formula is as follows:

$$
\mathrm{PRS}=\beta_{1} x_{1}+\beta_{2} x_{2}+\ldots \beta_{k} x_{k}+\beta_{n} x_{n}
$$

where $\beta \mathrm{k}$ is the per-allele OR for breast cancer associated with the minor allele for SNP $k$, and $x k$ is the number of alleles for the same $\operatorname{SNP}(0,1$, or 2$)$.

\section{Result}

\section{Disorders of estrogen expression in breast cancer patients}

Using LC-MS/MS quantitative analysis, we measured the expression levels of 11 serum estrogens and metabolites in 64 patients with premenopausal BC (mean age: $45.5 \pm 5.04$ years) and 49 matched controls (mean age: $43.7 \pm 8.80$ years). We found that there was no significant difference in age between the $\mathrm{BC}$ group and $\mathrm{NC}$ group. As shown in Fig. 1a, compared with the NC group, the BC group exhibited significantly increased estrogen levels, especially E1, E2, 2-OHE2, 4-OHE2 $(P<0.01)$ and $2 / 4-$ OHE1 $(P<0.05)$. OPLS-DA was constructed as an unsupervised statistical method to identify potential estrogen homeostatic changes between the two groups. As shown in Fig. 1b, the metabolic profile of the NC group was clearly separated from that of the $\mathrm{BC}$ group, indicating that there was a considerable metabolite difference between the $\mathrm{BC}$ group and $\mathrm{NC}$ group. We also found that the potential biomarkers with VIP values higher than 1.0 in the OPLS-DA model were E1, E2, 2OHE2, 4-OHE2 and 2/4-OHE1 in the serum of BC patients (Fig. 1c). Overall, these results supported the view that the disorder of estrogen homeostasis was closely related to increased risk of $\mathrm{BC}$.

\section{Cohort description and Hardy-Weinberg equilibrium testing}

We enrolled 140 patients first diagnosed with breast cancer and 140 corresponding healthy women in this study. The mean age at diagnosis (for patients with cancer) was $43.3 \pm 6.24$ years, and the mean age of healthy women at enrollment was $40.2 \pm 3.52$ years. Blood samples were collected from these participants to extract DNA and analyze the SNP genotype. We found that there was no significant difference in age between the $\mathrm{BC}$ group and NC group. The chi-square test was used to test the HWE value, and $P>0.05$ explained that the samples at enrollment were representative of the group. As seen in Table 2, all polymorphisms were found to be in genetic equilibrium, which indicated that the observed genotype frequencies of the case and control groups were constant and representative. 

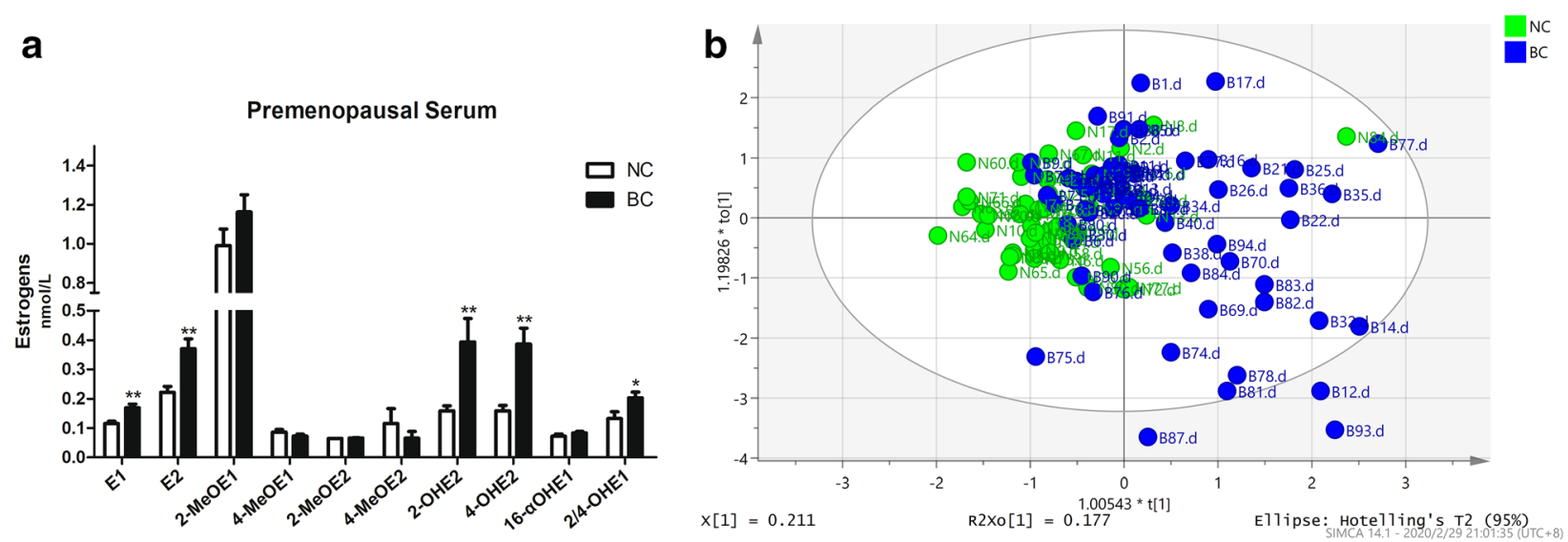

C VIP values of 11 estrogens in clinical premenopausal serum samples

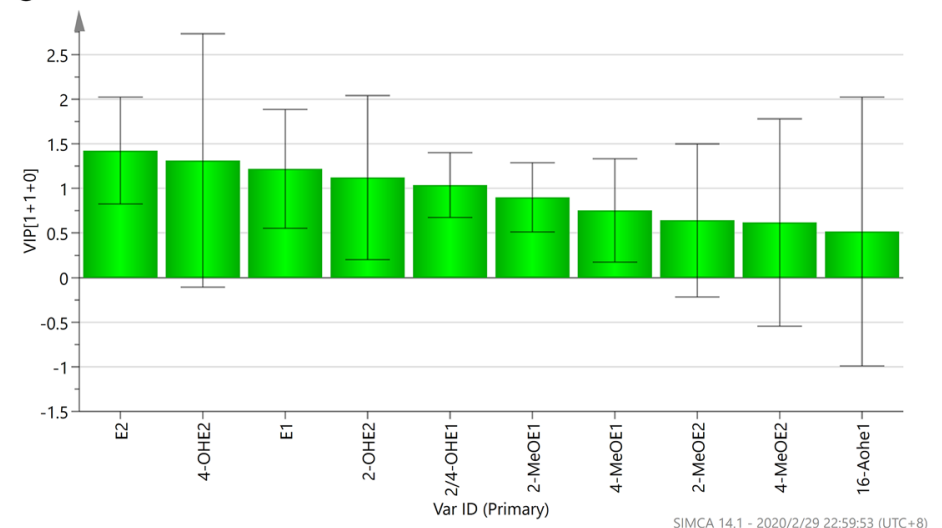

Fig. 1 Imbalance of estrogen homeostasis in the serum of BC patients. a The concentrations of estrogens, including estrone (E1), estradiol (E2), 16a-hydroxy estrone (16a-OHE1), 2-methoxy estrone (2-MeOE1), 4-methoxy estrone (4-MeOE1), 2-methoxy estradiol (2-MeOE2), 4-methoxy estradiol (4-MeOE2), 2/4-hydroxy estrone (2/4-OHE1), 2-hydroxy estradiol (2-OHE2), and 4-hydroxy estradiol (4-OHE2), in serum samples from NC ( 49 healthy women, mean age of $43.7 \pm 8.80$ years) and BC (64 breast cancer patients, mean age of $45.5 \pm 5.04$ years) were detected by LC-MS/MS. ${ }^{*}, p<0.05,{ }^{* *}, p<0.01$ vs control group. The results are shown as mean \pm SEM values to depict the levels of estrogens in serum of BC patients and healthy women. $\mathbf{b}$ Orthogonal Projections to Latent Structures-Discriminant Analysis (OPLS-DA) score plots of serum (R2X(cum) $=0.335$, $\mathrm{R} 2 \mathrm{Y}(\mathrm{cum})=0.264, \mathrm{Q} 2(\mathrm{cum})=0.003$ ) estrogen metabolites in NC group (green) and BC group (blue) generated by SIMCA 14.0 software. c Variable importance in the projection (VIP) values calculated from OPLS-DA models for estrogen metabolic profile data

\section{Association of estrogen-metabolizing enzyme genetic variants with breast cancer risk}

Table 3 shows univariate analysis and ORs related to each metabolizing enzyme SNP. The polymorphic genotypes of CYP1A1 rs1048943 $(P=0.007)$, CYP1B1 rs1056827 $(P=$ 0.004), CYP1B1 rs1056836 $(P=0.002)$ and SULT1A1 rs1042028 $(P=0.029)$ showed significant differences in distribution. Compared with the wild-type genotypes of CYP1A1 rs1048943 (TT) or SULT1A1 rs1042028 (CC), the heterozygous variant genotypes of CYP1A1 rs1048943 (TC) or SULT1A1 rs1042028 (CT) showed significantly higher risk in breast cancer, with ORs of 2.37 (95\% confidence interval $[\mathrm{CI}]=1.27-4.43)$ and $2.21(95 \% \mathrm{CI}=1.20$ 4.05), respectively. Compared with the wild-type genotypes of CYP1B1 rs1056827 (CC), the homozygous variant genotypes (AA) showed a significantly higher risk in breast cancer, yielding an OR of $6.90(95 \% \mathrm{CI}=1.50$
31.76). Compared with the wild-type genotypes of CYP1B1 rs1056836 (GG), the heterozygous variant genotypes significantly reduced the risk of breast cancer, yielding an OR of 0.37 (95\% CI $=0.21-0.67)$. In addition, no associations with breast cancer risk were observed for the estrogen metabolic enzyme gene SNPs CYP19A1 (rs700519), HSD17B1 (rs605059), COMT (rs4680), or UGT2B7 (rs7439366) or the GWAS-selected SNPs ZNF365 (rs10822013), FGFR2 (rs2981579), RAD51B (rs3784099), TOX3 (rs3803662), MAP3K1 (rs889312), or HCN1 (rs981782).

\section{PRS breast cancer risk prediction model establishment and evaluation}

The binary logistic regression method was used to calculate the OR of the per-allele model, and the detailed results are shown in Table 4. We used SNPs 
Table 3 Genotype frequencies and ORs associated with each gene polymorphism in breast cancer cases and controls

\begin{tabular}{|c|c|c|c|c|c|c|}
\hline Gene and SNPs & Genotype & Control n (\%) & Case $\mathrm{n}(\%)$ & $P$-value ${ }^{\#}$ & OR $(95 \% \mathrm{Cl})$ & $P$-value ${ }^{*}$ \\
\hline \multirow[t]{3}{*}{ CYP19A1 (rs700519) } & GG & $97(69.3 \%)$ & $92(65.7 \%)$ & 0.813 & 1 & - \\
\hline & GA & 37 (26.4\%) & $41(29.3 \%)$ & & $1.17(0.69-1.98)$ & 0.564 \\
\hline & AA & $6(4.3 \%)$ & $7(5.0 \%)$ & & $1.23(0.40-3.80)$ & 0.719 \\
\hline \multirow[t]{3}{*}{ CYP1A1 (rs1048943) } & $\pi$ & 100 (71.4\%) & $80(57.1 \%)$ & 0.007 & 1 & - \\
\hline & $\mathrm{TC}$ & $31(22.2 \%)$ & $55(39.3 \%)$ & & $2.37(1.27-4.43)$ & 0.003 \\
\hline & CC & $9(6.4 \%)$ & $5(3.6 \%)$ & & $1.10(0.30-4.00)$ & 0.528 \\
\hline \multirow[t]{3}{*}{ CYP1A1 (rs4649903) } & AA & $68(48.6 \%)$ & $58(41.4 \%)$ & 0.300 & 1 & - \\
\hline & $A G$ & $56(40.0 \%)$ & $58(41.4 \%)$ & & $1.21(0.73-2.02)$ & 0.453 \\
\hline & GG & $16(11.4 \%)$ & $24(17.1 \%)$ & & $1.76(0.85-3.62)$ & 0.126 \\
\hline \multirow[t]{3}{*}{ CYP1B1 (rs1056827) } & CC & $92(65.7 \%)$ & $80(57.1 \%)$ & 0.004 & 1 & - \\
\hline & CA & 48 (34.3\%) & 50 35.7\%) & & $1.20(0.73-1.97)$ & 0.802 \\
\hline & AA & $0(0.0 \%)$ & $10(7.2 \%)$ & & $6.90(1.50-31.76)$ & 0.001 \\
\hline \multirow[t]{3}{*}{ CYP1B1 (rs1056836) } & GG & $90(64.3 \%)$ & $116(82.9 \%)$ & 0.002 & 1 & - \\
\hline & GC & $44(31.4 \%)$ & $21(15.0 \%)$ & & $0.37(0.21-0.67)$ & 0.001 \\
\hline & CC & $6(4.3 \%)$ & $3(2.1 \%)$ & & $0.39(0.10-1.59)$ & 0.189 \\
\hline \multirow[t]{3}{*}{ HSD17B1 (rs605059) } & GG & 47 (33.6\%) & $46(32.9 \%)$ & 0.713 & 1 & - \\
\hline & GA & $73(52.1 \%)$ & $69(49.3 \%)$ & & $0.97(0.57-1.63)$ & 0.896 \\
\hline & $\mathrm{AA}$ & $20(14.3 \%)$ & $25(17.8 \%)$ & & $1.28(0.63-2.61)$ & 0.502 \\
\hline \multirow[t]{3}{*}{ COMT (rs4680) } & GG & $91(65.0 \%)$ & $80(57.1 \%)$ & 0.402 & 1 & - \\
\hline & GA & $42(30.0 \%)$ & $51(36.4 \%)$ & & $1.38(0.83-2.29)$ & 0.212 \\
\hline & AA & $7(5.0 \%)$ & $9(6.4 \%)$ & & $1.46(0.52-4.11)$ & 0.470 \\
\hline \multirow[t]{3}{*}{ SULT1A1 (rs1042028) } & $\mathrm{CC}$ & 117 (83.6\%) & $98(70.0 \%)$ & 0.029 & 1 & - \\
\hline & $\mathrm{CT}$ & $20(14.3 \%)$ & 37 (26.4\%) & & $2.21(1.20-4.05)$ & 0.010 \\
\hline & $\Pi$ & $3(2.1 \%)$ & $5(3.6 \%)$ & & $1.99(0.46-8.54)$ & 0.354 \\
\hline \multirow[t]{3}{*}{ UGT2B7 (rs7439366) } & CC & 69 (49.30\%) & $64(45.7 \%)$ & 0.824 & 1 & - \\
\hline & $\mathrm{CT}$ & 60 (42.80\%) & $65(46.4 \%)$ & & $1.17(0.72-1.90)$ & 0.533 \\
\hline & $\pi$ & $11(7.90 \%)$ & $11(7.90 \%)$ & & $1.08(0.44-2.66)$ & 0.870 \\
\hline \multirow[t]{3}{*}{ ZNF365 (rs10822013) } & CC & 36 (25.71\%) & $43(30.71 \%)$ & 0.640 & 1 & - \\
\hline & $C T$ & 75 (53.57\%) & $71(50.71 \%)$ & & $0.79(0.46-1.37)$ & 0.407 \\
\hline & $\pi$ & 29 (20.71\%) & $26(18.57 \%)$ & & $0.75(0.38-1.50)$ & 0.415 \\
\hline \multirow[t]{3}{*}{ FGFR2 (rs2981579) } & GG & 47 (33.57\%) & $40(28.57 \%)$ & 0.418 & 1 & - \\
\hline & GA & 70 (50.00\%) & 69 (49.29\%) & & $1.16(0.68-1.98)$ & 0.592 \\
\hline & AA & $23(16.43 \%)$ & $31(22.14 \%)$ & & $1.58(0.80-3.14)$ & 0.188 \\
\hline \multirow[t]{3}{*}{ RAD51B (rs3784099) } & GG & 111 (79.29\%) & $109(77.86 \%)$ & 0.848 & 1 & - \\
\hline & GA & $25(17.86 \%)$ & $28(20.00 \%)$ & & $1.14(0.63-2.08)$ & 0.668 \\
\hline & AA & $4(2.86 \%)$ & $3(2.14 \%)$ & & $0.76(0.17-3.49)$ & 0.728 \\
\hline \multirow[t]{3}{*}{ TOX3 (rs3803662) } & GG & 15 (10.71\%) & $18(12.86 \%)$ & 0.664 & 1 & - \\
\hline & GA & $61(43.57 \%)$ & $54(38.57 \%)$ & & $0.83(0.51-1.38)$ & 0.475 \\
\hline & AA & $64(45.71 \%)$ & $68(48.57 \%)$ & & $1.13(0.53-2.43)$ & 0.755 \\
\hline \multirow[t]{3}{*}{ MAP3K1 (rs889312) } & CC & 42 (30.00\%) & $35(25.00 \%)$ & 0.460 & 1 & - \\
\hline & CA & 67 (47.86\%) & $66(47.14 \%)$ & & $1.18(0.67-2.08)$ & 0.560 \\
\hline & $\mathrm{AA}$ & 31 (22.14\%) & 39 (27.86\%) & & $1.51(0.79-2.89)$ & 0.215 \\
\hline
\end{tabular}


Table 3 Genotype frequencies and ORs associated with each gene polymorphism in breast cancer cases and controls (Continued)

\begin{tabular}{|c|c|c|c|c|c|c|}
\hline Gene and SNPs & Genotype & Control n (\%) & Case n (\%) & $P$-value ${ }^{\#}$ & OR $(95 \% \mathrm{Cl})$ & $P$-value* \\
\hline \multirow[t]{3}{*}{ HCN1 (rs981782) } & CC & $16(11.43 \%)$ & $25(17.86 \%)$ & 0.475 & 1 & - \\
\hline & CA & 69 (49.29\%) & $63(45.00 \%)$ & & $0.92(0.55-1.53)$ & 0.737 \\
\hline & $\mathrm{AA}$ & 55 (39.29\%) & $52(37.14 \%)$ & & $1.45(0.68-3.08)$ & 0.336 \\
\hline
\end{tabular}

Values are presented as number (\%) or OR $(95 \% \mathrm{Cl})$

OR Odds radio, $\mathrm{Cl}$ Confidence interval, SNP Single nuclear polymorphism

\#Comparison of polymorphic genotype distributions in patients with breast cancer and healthy case-controls

*Comparison of wild-type genotypes with heterozygous genotypes and homozygous variant genotypes respectively

in the GWAS-identified high breast risk genes, namely, ZNF365 (rs10822013), FGFR2 (rs2981579), RAD51B (rs3784099), TOX3 (rs3803662), MAP3K1 (rs889312), and HCN1 (rs981782), to create PRS model 1 (M1) in the per-allele model. On the basis of M1, we also added estrogen metabolic enzyme gene SNPs, namely, CYP1A1 (rs1048943), CYP1B1 (rs1056827), SULT1A1 (rs1042028), CYP19A1 (rs700519), COMT (rs4680), HSD17B1 (rs605059), and UGT2B7 (rs7439366), to create PRS model 2 (M2). For SNPs in strong linkage disequilibrium located on the same gene or chromosome, we chose the one variant (rs1048943) with the lowest $P$ value in CYP1A1, and rs1056836 is a protective gene loci, we chose the risk variant rs10526827 in CYP1B1. The PRS scores are expressed as the means \pm SEM to find the difference between the two groups. Under M1 and M2, the PRS data of the two groups obeyed a normal distribution; therefore, we used an independent sample t-test to evaluate the difference between the two groups of data. As shown in Table 5 and Fig. 2, the PRS scores in the NC group were

Table 4 Univariate analysis and ORs associated with Per-allele model

\begin{tabular}{|c|c|c|c|c|}
\hline $\begin{array}{l}\text { Gene } \\
\text { name }\end{array}$ & $\begin{array}{l}\text { SNP rs } \\
\text { number }\end{array}$ & $\begin{array}{l}\text { Allele } \\
\text { Risk/ } \\
\text { reference }\end{array}$ & $\begin{array}{l}\mathrm{OR}^{\mathrm{a}}(95 \% \mathrm{Cl}) \\
\text { Per-allele }\end{array}$ & $\begin{array}{l}P \\
\text { value }^{*}\end{array}$ \\
\hline CYP19A1 & rs700519 & $\mathrm{G} / \mathrm{A}$ & $1.15(0.75-1.77)$ & 0.515 \\
\hline CYP1A1 & rs1048943 & $\mathrm{C} / \mathrm{T}$ & $1.43(0.94-2.16)$ & 0.094 \\
\hline CYP1B1 & rs1056827 & $\mathrm{A} / \mathrm{C}$ & $1.61(1.07-2.43)$ & $0.023^{*}$ \\
\hline HSD17B1 & rs605059 & $\mathrm{G} / \mathrm{A}$ & $1.09(0.78-1.53)$ & 0.607 \\
\hline COMT & rs4680 & $\mathrm{G} / \mathrm{A}$ & $1.31(0.88-1.90)$ & 0.188 \\
\hline SULT1A1 & rs1042028 & $\mathrm{T} / \mathrm{C}$ & $1.97(1.18-3.29)$ & $0.009^{*}$ \\
\hline UGT2B7 & rs7439366 & $\mathrm{T} / \mathrm{C}$ & $1.09(0.76-1.56)$ & 0.645 \\
\hline ZNF365 & rs10822013 & $\mathrm{C} / \mathrm{T}$ & $0.87(0.62-1.21)$ & 0.396 \\
\hline FGFR2 & rs2981579 & $\mathrm{G} / \mathrm{A}$ & $1.24(0.89-1.74)$ & 0.202 \\
\hline RAD51B & rs3784099 & $\mathrm{G} / \mathrm{A}$ & $1.03(0.62-1.72)$ & 0.896 \\
\hline TOX3 & rs3803662 & $\mathrm{G} / \mathrm{A}$ & $0.98(0.69-1.40)$ & 0.928 \\
\hline MAP3K1 & rs889312 & C/A & $1.00(0.72-1.39)$ & 1.000 \\
\hline HCN1 & rs981782 & $\mathrm{G} / \mathrm{A}$ & $1.20(0.85-1.69)$ & 0.297 \\
\hline
\end{tabular}

*Comparison in Per-allele model significantly lower than those in the $\mathrm{BC}$ group in M2 $\left(P=4.9^{*} 10^{-5}\right)$; however, there was no significant difference between $\mathrm{NC}$ and $\mathrm{BC}$ in $\mathrm{M} 1 \quad(P=0.17)$. Finally, the ROC curve was calculated to evaluate how the risk models discriminated between women with and without breast cancer (Fig. 3). The ROC curve estimated for M2 was 62.18\% (95\% confidence interval $[\mathrm{CI}]=0.56-0.69)$, whereas that for M1 was only $54.56 \%$ (95\% confidence interval $[\mathrm{CI}]=0.48-$ $0.61)$. Therefore, the accuracy of M2 in breast cancer risk identification was better than that of M1.

\section{Discussion}

Breast cancer $(\mathrm{BC})$ is an estrogen-dependent tumor, and the occurrence of $\mathrm{BC}$ is closely related to the imbalance of estrogen homeostasis. The accumulation of estrogen and its toxic metabolites in vivo is a significant risk factor for BC development. Different types of estrogens have different physiological and pathological activities and can play an important role in the process of cancer development through different mechanisms. Parent estrogens are postulated to promote tumorigenesis directly through the stimulation of the estrogen receptor (ER) [23]. The endogenous conversion of estrogen to genotoxic metabolites has been reported as an alternative, potentially ER-independent mechanism for estrogen-dependent breast tumorigenesis [24]. Catechol estrogens can form adducts with DNA, causing gene mutations and producing direct genotoxicity [13]. Methoxyestrogens, including 2-methoxyestradiol, have been shown to inhibit carcinogenesis by suppressing cell proliferation and estrogen oxidation due to their effects on microtubule stabilization [25].

In this study, the LC-MS/MS quantitative analysis method was used to determine the serum estrogens in the $\mathrm{BC}$ group and $\mathrm{NC}$ group. Comparing the levels of serum estrogens in the follicular phase and luteal phase of premenopausal breast cancer patients with healthy female volunteers, we found that the levels of parent and hydroxylated estrogen in the $\mathrm{BC}$ group were significantly higher than those in the NC group, which indicated that estrogen metabolism disorder is closely related to the occurrence and development of breast cancer. Using OPLS-DA, we also noticed that E1, E2, 4-OHE2, 2- 
Table 5 PRS value results and difference analysis of two gene combinations (M1 and M2)

\begin{tabular}{|c|c|c|c|c|c|}
\hline Model & Group & PRS (Mean \pm SEM) & Data distribution & Testing method & $P$ value \\
\hline \multirow[t]{2}{*}{ M1 } & NC group & $4.52 \pm 0.15$ & Normal distribution & T-test & 0.17 \\
\hline & BC group & $4.80 \pm 0.14$ & & & \\
\hline \multirow[t]{2}{*}{ M2 } & NC group & $8.38 \pm 0.21$ & Normal distribution & T-test & $4.90 * 10^{-5}$ \\
\hline & BC group & $9.63 \pm 0.22$ & & & \\
\hline
\end{tabular}

OHE2, and 2/4-OHE1 are BC-related disease markers. This result was consistent with the epidemiologic characteristics of patients with $\mathrm{BC}[26]$.

A large number of studies have confirmed that breast cancer exhibits heritability [27, 28]. However, high-risk genes such as BRCA1 and BRCA2 account for less than $15 \%$ of breast cancer cases $[29,30]$, which suggests that numerous breast cancer-related risk genes have not been discovered, and these gene polymorphisms influence susceptibility to breast cancer.

Estrogen is an important risk factor for breast cancer. However, no research has incorporated estrogens into the breast cancer risk prediction model. A possible major reason is that there is no clinically effective estrogen evaluation method because the steady state of estrogen is affected by various physiological and pathological factors, such as menstrual cycle fluctuations. However, estrogen homeostasis is regulated by various metabolic enzymes. Therefore, we believe that estrogen metabolic enzyme gene polymorphisms are closely related to estrogen homeostasis and the occurrence and development of breast cancer. In this study, univariate logistic regression analysis showed that CYP1A1, CYP1B1, and SULT1A1 gene polymorphisms are closely related to the occurrence of breast cancer. It is worth noting that these gene polymorphisms are also associated with other estrogendependent tumors such as endometrial cancer and ovarian cancer. Hiroshi Hirata et al. found that the SULT1A1

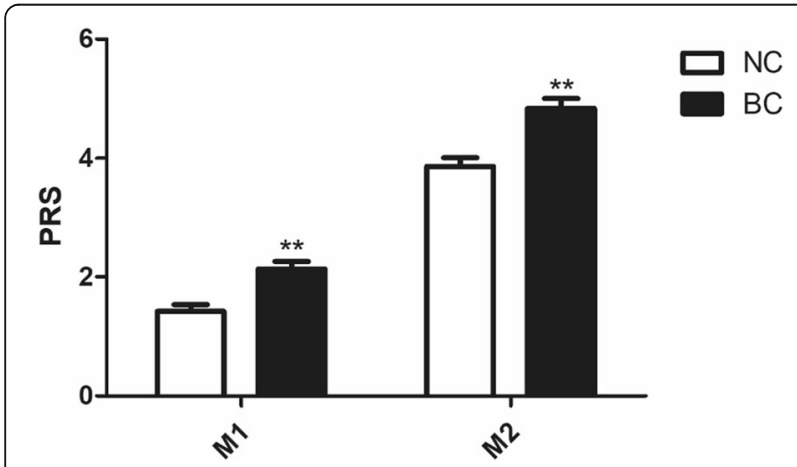

Fig. 2 The Polygenic Risk Scores (PRS) of the NC group and BC group in the two risk gene models: PRS model 1 (M1) and PRS model 2 (M2). The results are shown as mean \pm SEM values to depict the distribution difference between $\mathrm{NC}$ and $\mathrm{BC} .{ }^{*}, p<0.05$, **, $p<0.01$ vs control group rs9282861 (rs1042028) was related to endometrial cancer [31]. A meta-analysis was performed to research the association between CYP1A1 gene polymorphism and ovarian cancer risk, which showed that the Ile/Val (rs1048943) was significantly associated with ovarian cancer, with homozygous carriers (Val/Val vs. Ile/Ile: $\mathrm{OR}=2.64 ; 95 \% \mathrm{CI}: 1.63-4.28$ ) being risk factors for ovarian cancer development [32].

CYP1A1 and CYP1B1 are the major phase I drug metabolism enzymes that catalyze the hydroxylation of estrogens. The increasing polarity of estrogens may be related to the risk of breast cancer [14]. Our experiments also verified this view. In this study, we found that the variant allele of CYP1B1 rs1086836 was involved in reducing the risk of breast cancer and that the exact mechanism of the protection of this variant allele was not clear [33]. We assumed that the heterozygous model of CYP1B1 rs1086836 (GC vs. GG: OR $=0.37,95 \% \mathrm{CI}$ : $0.21-0.67, P=0.001)$ may result in decreased function of the CYP1B1 enzyme, reducing the production of 4hydroxy estrogen and even catechol estrogen-3,4-quinone (CE-3,4-Q) to form adducts with DNA. At the same time, this study also proved that the variant alleles of CYP1A1 rs1048943 (TC vs. TT: OR $=2.37$, 95\% CI: 1.27-4.43, $P=0.003$ ) and CYP1B1 rs1056827 (AA vs. CC: $\mathrm{OR}=6.90,95 \% \mathrm{CI}: 1.50-31.76, P=0.001$ ) are closely related to the risk of breast cancer, which is consistent with most research $[34,35]$. The possible reason is that the mutations promote the activity of CYP1A1 and CYP1B1 enzymes to increase the production of hydroxylated estrogens or promote the individual's susceptibility to estrogen.

SULTs catalyze the sulfate conjugation of a broad range of substrates and play an important role in the metabolism of endogenous and exogenous compounds, including thyroid and steroid hormones, neurotransmitters, drugs and procarcinogens [36]. SULTs catalyze the sulfated metabolism of estrogen (E1 and E2) and its metabolites (such as catechol estrogen) and eliminate the activity of estrogen by forming sulfate compounds: sulfated estrogens that cannot combine with estrogen receptors (ERs). At the same time, it promotes the rapid excretion of sulfated estrogen from the cells, which can reduce the level of estrogen exposure in the circulation and target tissues. SULT1A1 rs1042028 is the most widely studied gene polymorphism. Its allelic variation 


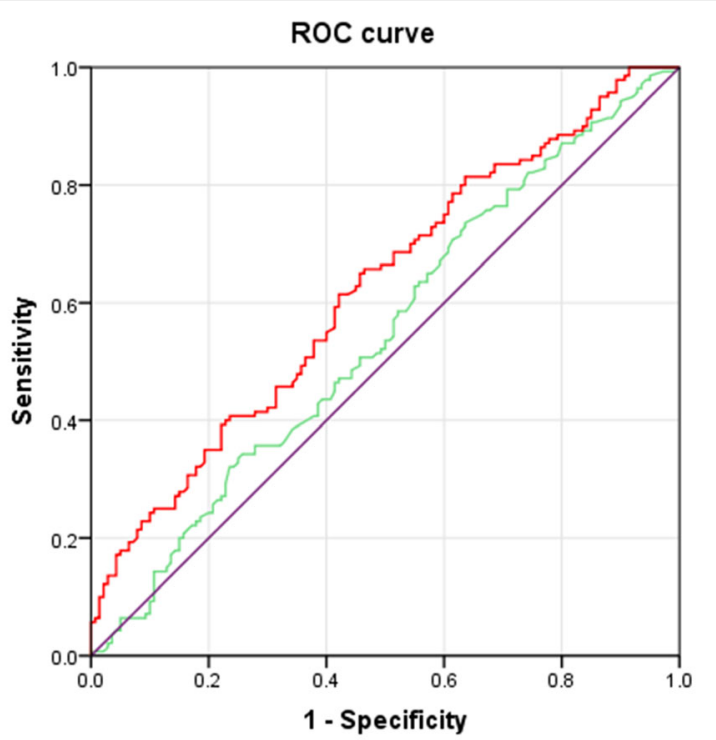

— PRS model 1 (M1): rs1 0822013,rs2981579,rs3784099,rs3803662,rs889312,rs981782

— PRS model 2 (M2): rs10822013,rs2981579,rs 3784099, ,rs 3803662, rs 889312, ,rs 981782, rs 1042028 ,

- Reference rs1048943,rs1056827,rs4680,rs605059,rs700519,rs7439366

Fig. 3 The receiver operating characteristic (ROC) curve in the two risk models. The purple line with an area under the ROC curve (AUC) of 50\% is the reference. The AUC of the upper red line, which showed the PRS model 2 (M2), is $62.18 \%$. The green line with a ROC of $54.56 \%$ is PRS model 1 (M1)

can reduce enzyme activity and thermal stability, resulting in increased estrogen accumulation and increased individual susceptibility to breast cancer [37]. In this study, the heterozygous model of rs1042028 had a 2.21fold higher risk of breast cancer than the wild-type model. This is consistent with the results of multiple studies [38, 39].

Previous studies investigated associations between the PRS of multiple SNPs and breast cancer risk to study the cumulative effect of genes on the disease. Mavaddat et al. constructed a 77-SNP PRS for breast cancer and found a threefold increase in risk when comparing the polygenic scores of the highest $1 \%$ and the middle quintiles [9]. Harlid et al. investigated the combined effect of low-penetrant SNPs on breast cancer, which included ten SNPs, and found that the cumulative effect was strongly correlated with breast cancer [40]. However, most of this research on PRS comes from the Caucasian population sample database. Although Sueta, Chan and others have also conducted similar studies in Asian populations, the evidence is still limited [7, 41]. To date, there have been no relevant reports on the establishment of a breast cancer PRS risk prediction model from the perspective of estrogen-metabolizing enzymes. Thus, a multigene PRS model including estrogen metabolic enzyme gene SNPs and GWAS-selected SNPs was constructed in this study to evaluate the comprehensive effects of multiple estrogen metabolic enzyme SNPs on breast cancer.
In this study, we evaluated possible relationships between the increased breast cancer risk estrogen metabolic enzyme gene SNPs and GWAS-identified gene SNPs in an Asian population. Among them, the GWASidentified SNPs were not associated with breast cancer risk in the per-allele model or dominant model in our study. This finding was inconsistent with a previous study [23]. Further, we established PRS model 1, including only GWAS-identified SNPs, and PRS model 2, which included estrogen metabolic enzyme gene SNPs on the basis of M1. By calculating the PRS score of each individual under the M1 and M2 PRS models and performing a t-test analysis on the PRS score of the $\mathrm{BC}$ and NC groups, we found that the $P$-value $\left(4.9^{*} 10^{-5}\right)$ of the M2 PRS model was far less than that of M1 (0.17). Moreover, the ROC curve $(62.18 \%)$ of the M2 model was better than that of the M1 model (54.56\%). Therefore, the model constructed by adding estrogen metabolic enzyme gene SNPs had a good ability in breast cancer risk prediction, and the accuracy was greatly improved.

There are several limitations of this study that should be noted. First, the sample size was relatively small. In this study, only 140 premenopausal women first diagnosed with BC and 140 matched healthy women were recruited based on our criteria; thus, we did not have enough statistical power to detect the effect of the genetic variants on some of the parameters. Second, because funding was limited, it did not include comprehensive 
metabolic enzymes and adequate breast cancer risk gene loci. Due to these reasons, the AUC was small and the model have not been tested. In the future, we will study additional estrogen-metabolizing enzyme genes and other breast cancer risk genes in our research. At the same time, we will also include recognized breast cancer risk factors such as age at evaluation, age at menarche, age at first live birth, race, number of breasts, and family history of breast cancer and construct a breast cancer risk prediction model composed of phenotype and genotype to obtain a more valuable ROC value. In addition, the sample size needs to be further expanded, and it is better to include more data information of different races.

\section{Conclusion}

Estrogens and related metabolic enzyme gene polymorphisms are closely related to $\mathrm{BC}$. The model constructed by adding estrogen metabolic enzyme gene SNPs has good predictive ability for breast cancer risk, and the accuracy is greatly improved compared with that of the PRS model that only includes GWAS-identified gene SNPs.

\begin{abstract}
Abbreviations
BC: Breast cancer; BMI: Body mass index; Cl: Confidence interval; COMT: Catechol-O-methyltransferase; CYP: Cytochrome P450; E1: Estrone; E2: 17ß-estradiol; 2-OHE2/1: 2-hydroxy estradiol/estrone; 4-OHE2/1: 4-hydroxy estradiol/estrone; 16a-OHE1: 16a-hydroxy estrone; 2-MeOE2/1: 2-methoxy estradiol/estrone; 4-MeOE2/1: 4-methoxy estradiol/estrone; ER: Estrogen receptor; ESI: Electrospray ionization source; FRR: Familial relative risk; GWAS: Genome-wide association study; HWE: Hardy-Weinberg equilibrium; LC-MS/MS: Liquid chromatography-tandem mass spectrometry; M1: PRS model 1; M2: PRS model 2; MRM: Multiple reaction monitoring; OPLSDA: Orthogonal Partial Least Squares Discriminant Analysis; OR: Odds ratio; PRS: Polygenic risk score; QC: Quality control; ROC: Receiver operating characteristic curve; SNPs: Single nucleotide polymorphisms; SULTs: Sulfate transferases; UGTs: UDP-glucuronosyl-transferases; WHO: World Health Organization
\end{abstract}

\section{Acknowledgments}

Not applicable.

\section{Authors' contributions}

All authors read and approved the final manuscript. XYZ, YL and FZ contributed to conception and design. $F Z, X Y Z$, and $Z X H$ performed the methodology. FZ, ZXH and YNZ collected experimental data. FZ and ZXH analyzed and explained the data. FZ and XYZ wrote, reviewed, and revised the manuscript. YXX, BZ, MG, and XXY contributed to administrative, technical, and material support. XYZ supervised the research. All authors read and approved the final manuscript.

\section{Funding}

This study was supported by the Natural Science Foundation of the Jiangsu Higher Education Institutions of China [No. 18KJA350002]; the Natural Science Foundation of Jiangsu Province [No. BK20181470]; the Science and Technology Foundation of Xuzhou [No. KC18044]; the Natural Science Foundation of China [No. 81403001]; the Six Talent Peaks Project in Jiangsu Province [YY-045]; the Qing Lan Project in Jiangsu Province; the Provincial Commission of Health and Family Planning in Jiangsu Province [No. H2017079]; the Natural Science Foundation General Project of Jiangsu Province [No. BK20171173]; and the Science and Technology Planning Project of Jiangsu Province [No. BE2019636]. The roles are to provide essential funding to pay personal efforts.

\section{Availability of data and materials}

The data that support the findings of this study are available from the corresponding author upon reasonable request.

\section{Ethics approval and consent to participate}

All procedures performed in studies involving human participants followed the ethical standards of the institutional and/or national research committee and complied with the 1964 Helsinki Declaration and its later amendments or comparable ethical standards. This study is registered on the Clinical Test Public Management Platform (Registration number: ChiCTR1800014658). The protocol was approved by the Ethics Committee of the Affiliated Hospital of Xuzhou Medical University (XYFY2017-KL008-01). Informed consent was obtained from all individual participants included in the study.

\section{Consent for publication}

Not applicable.

\section{Competing interests}

The authors declare that they have no competing interests.

\section{Author details}

'Jiangsu Key Laboratory of New Drug Research and Clinical Pharmacy, College of Pharmacy, Xuzhou Medical University, 209 Tongshan Road, Xuzhou 221004, China. ${ }^{2}$ Department of Pharmacy, The First People's Hospital of Yancheng, The Yancheng Clinical College of Xuzhou Medical University, Yancheng, China. ${ }^{3}$ Department of Thyroid and Breast Surgery, The Affiliated Hospital of Xuzhou Medical University, Xuzhou, China. ${ }^{4}$ Department of Obstetrics and Gynecology, Xuzhou Central Hospital, Xuzhou Clinical School of Xuzhou Medical University, Xuzhou, China.

Received: 2 August 2020 Accepted: 9 February 2021

Published online: 25 February 2021

\section{References}

1. Heer E, Harper A, Escandor N, Sung H, McCormack V, Fidler-Benaoudia MM. Global burden and trends in premenopausal and postmenopausal breast cancer: a population-based study. Lancet Glob Health. 2020;8(8):e1027-37.

2. Muhammad N, Steele R, Isbell TS, Philips N, Ray RB. Bitter melon extract inhibits breast cancer growth in preclinical model by inducing autophagic cell death. Oncotarget. 2017:8(39):66226-36 Published 2017 Aug 3.

3. Mohammad N, Malvi P, Meena AS, et al. Cholesterol depletion by methyl- $\beta$ cyclodextrin augments tamoxifen induced cell death by enhancing its uptake in melanoma. Mol Cancer. 2014;13:204 Published 2014 Sep 1.

4. Gail MH, Brinton LA, Byar DP, et al. Projecting individualized probabilities of developing breast cancer for white females who are being examined annually. J Natl Cancer Inst. 1989;81(24):1879-86.

5. Crispo A, D'Aiuto G, De Marco M, et al. Gail model risk factors: impact of adding an extended family history for breast cancer. Breast J. 2008;14(3): 221-7.

6. Bonache S, Gutierrez-Enriquez S, Tenés A, Masas M, Balmaña J, Diez O, Mutation analysis of the BCCIP gene for breast cancer susceptibility in breast/ovarian cancer families. Gynecol Oncol. 2013;131(2):460-3.

7. Chan M, Ji SM, Liaw CS, et al. Association of common genetic variants with breast cancer risk and clinicopathological characteristics in a Chinese population. Breast Cancer Res Treat. 2012;136(1):209-20.

8. Möller S, Mucci LA, Harris JR, et al. The heritability of breast cancer among women in the Nordic Twin Study of Cancer. Cancer Epidemiol Biomark Prev. 2016;25(1):145-50.

9. Mavaddat N, Pharoah PD, Michailidou K, et al. Prediction of breast cancer risk based on profiling with common genetic variants. J Natl Cancer Inst. 2015;107(5):djv036.

10. Warren Andersen S, Trentham-Dietz A, Gangnon RE, et al. The associations between a polygenic score, reproductive and menstrual risk factors and breast cancer risk. Breast Cancer Res Treat. 2013;140(2):427-34.

11. Reeves GK, Travis RC, Green J, et al. Incidence of breast cancer and its subtypes in relation to individual and multiple low-penetrance genetic susceptibility loci. JAMA. 2010;304(4):426-34.

12. Tam V, Patel N, Turcotte M, Bossé Y, Paré G, Meyre D. Benefits and limitations of genome-wide association studies. Nat Rev Genet. 2019;20(8): 467-84. 
13. Warner M, Gustafsson JA. On estrogen, cholesterol metabolism, and breast cancer. N Engl J Med. 2014;370(6):572-3.

14. Zhang Y, Gaikwad NW, Olson K, et al. Cytochrome P450 isoforms catalyze formation of catechol estrogen quinones that react with DNA. Metabolism 2007;56:887-94

15. Kiruthiga PV, Kannan MR, Saraswathi C, et al. CYP1A1 gene polymorphisms: lack of association with breast cancer susceptibility in the southern region (Madurai) of India. Asian Pac J Cancer Prev. 2011;12:2133-8.

16. Crooke PS, Ritchie MD, Hachey DL, et al. Estrogens, enzyme variants and breast cancer: a risk model. Cancer Epidemiol Biomark Prev. 2006; 15:1620-9.

17. Ghisari $M$, Eiberg $H$, Long $M$, et al. Polymorphisms in phase I and phase $\|$ genes and breast cancer risk and relations to persistent organic pollutant exposure: a case-control study in Inuit women. Environ Health. 2014;13:19.

18. Qiu J, Du Z, Liu J, et al. Association between polymorphisms in estrogen metabolism genes and breast cancer development in Chinese women: a prospective case-control study. Medicine. 2018;97(47):e13337.0.

19. Sangrajrang S, Sato $Y$, Sakamoto $H$, et al. Genetic polymorphisms of estrogen metabolizing enzyme and breast cancer risk in Thai women. Int J Cancer. 2009:125(4):837-43.

20. Ghisari M, Long M, Røge DM, et al. Polymorphism in xenobiotic and estrogen metabolizing genes, exposure to perfluorinated compounds and subsequent breast cancer risk: a nested case-control study in the Danish National Birth Cohort. Environ Res. 2017;154:325-33.

21. Zhao F, Wang $X$, Wang $Y$, et al. The function of uterine UDPglucuronosyltransferase 1A8 (UGT1A8) and UDP-glucuronosyltransferase 2B7 (UGT2B7) is involved in endometrial cancer b ased on estrogen metabolism regulation. Hormones (Athens). 2020;19(3):403-12.

22. Hsieh YC, Tu SH, Su CT, et al. A polygenic risk score for breast cancer risk in a Taiwanese population. Breast Cancer Res Treat. 2017;163(1):131-8.

23. Eliassen AH, Spiegelman D, Xu X, Keefer LK, Veenstra TD, Barbieri RL, et al, Urinary estrogens and estrogen metabolites and subsequent risk of breast cancer among premenopausal women. Cancer Res. 2012;72:696-706.

24. Newbold RR, Liehr JG. Induction of uterine adenocarcinoma in CD-1 mice by catechol estrogens. Cancer Res. 2000;60:235-7.

25. Nehal J, Laldmni, Mohamadi A, et al. 2-Methoxyestradiol, a promising anticancer agent. Pharmacotherapy. 2003;23(2):165-72.

26. Sampson JN, Falk RT, Schairer C, et al. Association of estrogen metabolism with breast cancer risk in different cohorts of postmenopausal women. Cancer Res. 2017;77:918-25.

27. Blazer KR, Slavin T, Weitzel JN. Increased reach of genetic cancer risk assessment as a tool for precision management of hereditary breast cancer. JAMA Oncol. 2016;2:723-4.

28. Doherty J, Bonadies DC, Matloff ET. Testing for hereditary breast cancer: panel or targeted testing? Experience from a clinical cancer genetics practice. J Genet Counsel. 2015:24:683-7.

29. Bogdanova N, Helbig S, Dork T. Hereditary breast cancer: ever more pieces to the polygenic puzzle. Hered Cancer Clin Pract. 2013;11:12.

30. El Saghir NS, Zgheib NK, Assi HA, et al. BRCA1 and BRCA2 mutations in ethnic Lebanese Arab women with high hereditary risk breast cancer. Oncologist. 2015;20:357-64.

31. Hirata H, Hinoda Y, Okayama N, et al. CYP1A1, SULT1A1, and SULT1E1 polymorphisms are risk factors for endometrial cancer susceptibility. Cancer. 2008:112(9):1964-73.

32. Huang $M$, Chen $Q$, Xiao J, Zhao X, Liu C. CYP1A1 lle462Val is a risk factor for ovarian cancer development. Cytokine. 2012;58(1):73-8.

33. Gajjar K, Martin-Hirsch PL, Martin FL. CYP1B1 and hormone-induced cancer. Cancer Lett. 2012;324:13-30.

34. Martínez-Ramírez OC, Pérez-Morales R, Castro C, et al. Polymorphisms of catechol estrogens metabolism pathway genes and breast cancer risk in Mexican women. Breast. 2013;22:335-43.

35. Reding KW, Weiss NS, Chen C, et al. Genetic polymorphisms in the catechol estrogen metabolism pathway and breast cancer risk. Cancer Epidemiol Biomark Prev. 2009;18:1461-7

36. Xiao J, Zheng Y, Zhou Y, Zhang P, Wang J, Shen F, et al. Sulfotransferase SULT1A1 Arg213His polymorphism with cancer risk: a meta-analysis of 53 case-control studies. PLoS One. 2014;9(9):e106774.

37. Nagar S, Walther S, Blanchard RL. Sulfotransferase (SULT) 1A1 polymorphic variants *1, *2, and *3 are associated with altered enzymatic activity, cellular phenotype, and protein degradation. Mol Pharmacol. 2006;69:2084-92.
38. Lee H, Wang Q, Yang F, Tao P, Li H, Huang Y, et al. SULT1A1 Arg213His polymorphism, smoked meat, and breast cancer risk: a case-control study and meta-analysis. DNA Cell Biol. 2012;31(5):688-99.

39. Forat-Yazdi M, Jafari M, Kargar S, Abolbaghaei SM, Nasiri R, et al. Association between SULT1A1 Arg213His (rs9282861) polymorphism and risk of breast cancer: a systematic review and meta-analysis. J Res Health Sci. 2017;17(4): e00396.

40. Harlid S, Ivarsson MI, Butt S, et al. Combined effect of low-penetrant SNPS on breast cancer risk. Br J Cancer. 2012;106(2):389-96.

41. Sueta $A$, Ito $H$, Kawase $T$, et al. A genetic risk predictor for breast cancer using a combination of low-penetrance polymorphisms in a Japanese population. Breast Cancer Res Treat. 2012;132(2):711-21.

\section{Publisher's Note}

Springer Nature remains neutral with regard to jurisdictional claims in published maps and institutional affiliations.
Ready to submit your research? Choose BMC and benefit from:

- fast, convenient online submission

- thorough peer review by experienced researchers in your field

- rapid publication on acceptance

- support for research data, including large and complex data types

- gold Open Access which fosters wider collaboration and increased citations

- maximum visibility for your research: over $100 \mathrm{M}$ website views per year

At $\mathrm{BMC}$, research is always in progress.

Learn more biomedcentral.com/submissions 